\title{
Improved Assessment of Aortic Calcification in Japanese Patients Undergoing Maintenance Hemodialysis
}

\author{
Masaki Ohya ${ }^{1}$, Haruhisa Otani ${ }^{2}$, Keigo Kimura ${ }^{3}$, Yasushi Saika ${ }^{4}$, Ryoichi Fujii ${ }^{4}$, \\ Susumu Yukawa $^{5}$ and Takashi Shigematsu ${ }^{1}$
}

\begin{abstract}
Objective Vascular calcification is a feature of arteriosclerosis and in hemodialysis (HD) patients it may be severe, even at a relatively young age, and is closely related to the overall prognosis. We used the aortic calcification area index (ACAI), derived from the aortic calcification index (ACI), to evaluate and analyze the risk factors for abdominal aortic calcification in HD patients.

Patients and Methods Subjects comprised 137 patients on maintenance HD. ACAI was measured on abdominal plain computed tomography: 10 slices of the abdominal aorta were obtained at 1-cm intervals from the bifurcation of the common iliac artery and the area of the aortic cross-section and calcification was measured using image software. The calcification area was divided by the cross-sectional area and expressed as a percentage (\%). The mean value for the 10 slices was also calculated. Patients were divided into 2 groups according to ACAI being lower or higher than the mean value and the risk factors in each group were compared by multivariate analysis.

Results Group comparison showed significant differences in age, systolic blood pressure, serum calcium, and lipoprotein(a). On multiple regression analysis, age, systolic blood pressure, and serum calcium were independent risk factors. On logistic regression analysis, age, duration of dialysis, systolic blood pressure, and serum calcium were independent risk factors.

Conclusion Risk factors for abdominal aortic calcification in HD patients include age, systolic blood pressure, and serum calcium, according to ACAI evaluation. The ACAI was accurate and useful for evaluating abdominal calcification.
\end{abstract}

Key words: abdominal aorta, blood pressure, calcification, calcium, chronic kidney disease, hemodialysis

(Inter Med 49: 2071-2075, 2010)

(DOI: 10.2169/internalmedicine.49.3752)

\section{Introduction}

In patients with chronic kidney disease (CKD), especially CKD-5Dialysis(D) patients, the risk of cardiovascular disease is increased compared with the general population. Arteriosclerosis is closely associated with vascular calcification, which is often severe in patients on hemodialysis (HD), even when they are young (1).

Vascular calcification in HD patients is mainly intimal atherosclerosis or medial calcification (Mönckeberg's calcifi- cation). Atherosclerosis commonly involves the large and mid-sized muscular arteries, such as the aorta and coronary arteries, in which intimal lipid deposition, inflammatory cell infiltration (e.g. T-cell macrophages), and formation of plaques and raised lesions are seen. Mönckeberg's calcification occurs in the media of mid-sized and smaller peripheral arteries. It is not necessarily associated with lumen stenosis, but compliance decreases as vessel stiffness increases, resulting in elevated systolic blood pressure (BP), increased afterload, left ventricular hypertrophy, and increased myocardial oxygen demand. In addition, as diastolic BP de-

${ }^{1}$ Division of Nephrology and Blood Purification Medicine, Wakayama Medical University, Wakayama, ${ }^{2}$ Kisen Kidney Disease Clinic, Wakayama, ${ }^{3}$ Department of Internal Medicine, Sakura Clinic, Kainan, ${ }^{4}$ Department of Internal Medicine, Ryosyukai Fujii Hospital, Kishiwada and ${ }^{5}$ Department of Internal Medicine, Hakubunkai Kodama Hospital, Wakayama

Received for publication April 3, 2010; Accepted for publication July 12, 2010

Correspondence to Dr. Masaki Ohya, m-ohya@384.jp 

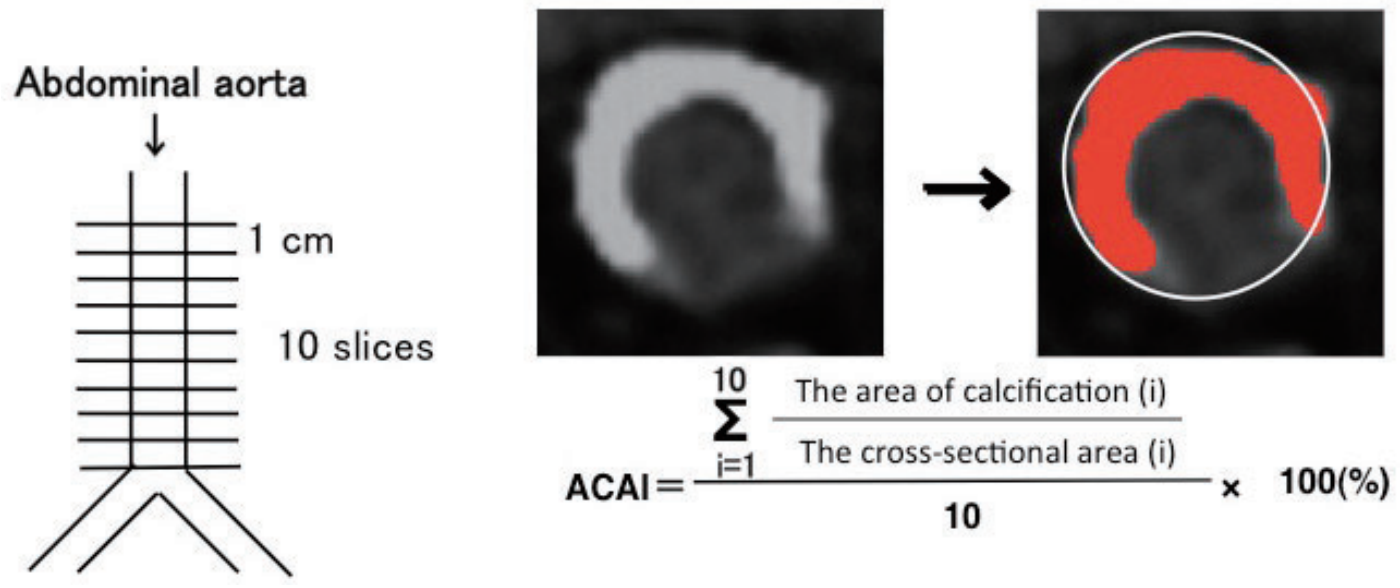

Figure 1. Calculation of aortic calcification area index (ACAI).

creases, coronary blood flow also decreases. These hemodynamic changes lead to ischemic heart disease. The cause of death in approximately $40 \%$ of HD patients is cardiovascular complications (2). With advances in dialysis technology and treatment, patients are living longer, and therefore it is becoming increasingly important to effectively evaluate and prevent the progression of vascular calcification.

Clinical evaluation of vascular calcification includes the identification of risk factors for the onset and progression of cardiovascular disease. Conventional means of diagnosing vascular calcification include detecting calcification in the aortic arch or common iliac artery on plain radiography $(3,4)$, aortic pulse-wave velocity (PWV) (5), and electron beam CT (EBCT) (6) or multidetector-row helical CT (MDCT) (7). Each of these modalities has advantages and disadvantages in terms of convenience, accuracy, and safety. PWV is useful for adjunctive diagnosis, but is not an imaging modality and is affected by hypertension. EBCT and MDCT are excellent for quantitative evaluation of coronary artery calcification, but evaluation can be difficult in patients with coronary stents, severe calcification or tachycardia, and in those who cannot hold their breath for more than $30 \mathrm{~s}$. In addition, because of the expensive equipment required, these studies are not available at many dialysis centers. Plain radiography is the most convenient and inexpensive, but does not provide quantitative information.

The aortic calcification index (ACI) is conventionally used to clinically evaluate abdominal aortic calcification (8). Using the imaging modality, 10 slices of the abdominal aorta are obtained at $1-\mathrm{cm}$ intervals from the common iliac bifurcation. The ACI score expresses calcification in $12 \mathrm{sec}-$ tors as a percentage (\%), so the extent of calcification in the aortic wall circumference is assessed, but does not correspond to the "thickness" of the calcification.

\section{Patients and Methods}

The study group comprised 137 patients (70 men, 67 women) on maintenance HD 3 times per week (HD duration
$>3$ months) at a single dialysis center in Japan for 3 years. Abdominal plain computed tomography (CT) was performed annually to exclude renal cell carcinoma and other malignant complications. During imaging, 10 slices of the abdominal aorta were obtained at $1-\mathrm{cm}$ intervals from the common iliac bifurcation. The area of each aortic crosssection and of calcification was measured using NIH Image software (NIH: National Institutes of Health, United State of America). The area of calcification was then divided by the aortic cross-sectional area and expressed as a percentage. ACAI was taken as the mean value of 10 slices (Fig. 1). Figure 2 shows a range of aortic calcification, for which the ACI scores are similar $(7 / 12=58.3 \%)$, whereas the ACAI scores, which directly measure the area of calcification, are not similar $(20.4 \%, 31.2 \%$, and $43.6 \%)$. Thus, in patients with the same ACI score, the ACAI is a more accurate evaluation of the status of aortic calcification.

Based on the extent of abdominal aortic calcification, patients were divided into a low $(\mathrm{L})$ or high $(\mathrm{H})$ group according to whether the ACAI was below or above the mean value. Blood tests for each patient were routinely performed at the start of dialysis and a total of 24 times per year. Assays were performed using a standard biochemical analyzer (Auto Biochemical Analyzer 7,170, Hitachi, Tokyo, Japan). Systolic and diastolic BP were measured 156 times per year and recorded as mean values for each patient before dialysis.

Data are expressed as mean \pm SD. The unpaired Student's t-test and Mann-Whitney U test were used to compare discrete variables between groups. Multivariate analysis was performed using multivariable linear regression and logistic regression analysis to identify independent risk factors for aortic calcification.

\section{Results}

Among the 137 patients, the ACAI ranged from $0 \%$ to $57.6 \%$, with a mean of $20.7 \pm 15.3 \%$; 9 patients had no abdominal aortic calcification (ACAI $=0 \%)$. 
ACI
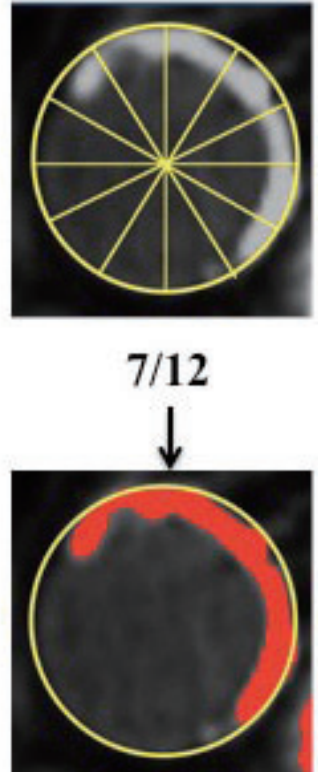

$20.4(\%)$
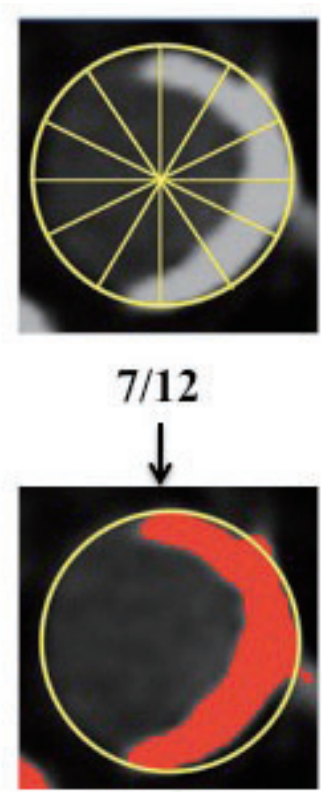

$31.2(\%)$
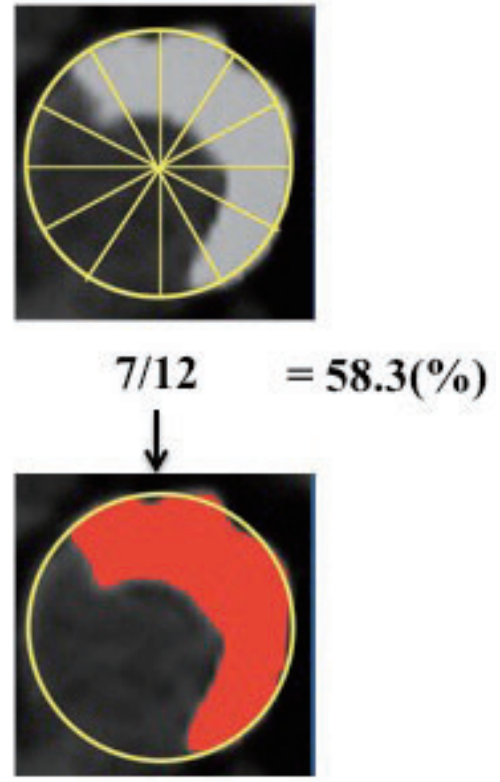

43.6(\%)

Figure 2. Comparison of aortic calcification index (ACI) and aortic calcification area index (ACAI).

Table 1. Baseline Characteristics of Hemodialysis Patients according to the Aortic Calcification Area Index (ACAI)

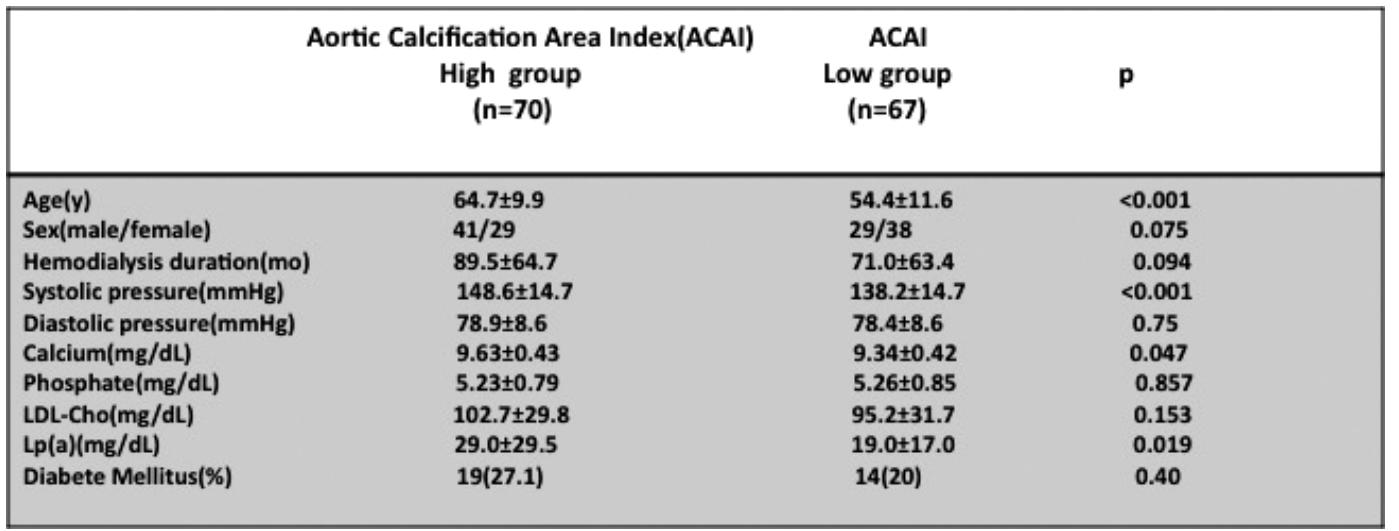

When the $\mathrm{H}$ and $\mathrm{L}$ groups were compared for risk factors, age, systolic BP, and serum calcium, and lipoprotein(a) (Lp(a) levels differed significantly between groups (Table 1).

Multivariate analysis was performed using multivariable linear regression and logistic regression analyses to identify independent risk factors for aortic calcification. In the final model $\left(\mathrm{R}^{2}=0.44\right)$, age, systolic and diastolic $\mathrm{BP}$, and serum calcium, and $\mathrm{Lp}$ (a) levels were independent risk factors for aortic calcification (Table 2).

The same factors were analyzed by logistic regression analysis and in the final model, age, duration of dialysis, systolic BP, and the serum calcium level were independent risk factors for aortic calcification (Table 3).

\section{Discussion}

Mild renal dysfunction and urinary abnormalities are known risk factors for cardiovascular disease (9), therefore HD patients have a 5- to 20 -fold higher risk than the general population and the major reason is early arteriosclerosis (10). Vascular calcification is often reported in HD patients, even at a young age, and the characteristic changes include intimal atherosclerosis and medial Mönckeberg's calcification. Clinical evaluation of vascular calcification includes the identification of risk factors for the onset and progression of cardiovascular disease.

Abdominal plain CT is available even in small and medium-sized dialysis centers. Because of the higher risk of renal cell carcinoma in HD patients, abdominal plain CT is 
Table 2. Multivariable Linear Regression Risk for Aortic Calcification

\begin{tabular}{|c|c|c|c|}
\hline & logACAl $\beta$ Coefficient & $95 \%$ Confidence interval & $\mathbf{p}$ \\
\hline \multicolumn{4}{|l|}{ Model 1: $\mathrm{R}^{2}=0.46(\mathrm{~N}=131)$} \\
\hline Age(y) & 0.53 & 0.34 to 0.73 & $<0.001$ \\
\hline Hemodialysis duration(mo) & 0.03 & 0.002 to 0.07 & 0.064 \\
\hline Systolic pressure $(\mathrm{mmHg})$ & 0.43 & 0.25 to 0.62 & $<0.001$ \\
\hline Diastolic pressure $(\mathrm{mmHg})$ & -0.44 & -0.796 to -0.08 & 0.02 \\
\hline Calcium(mg/dL) & 8.48 & 3.29 to 13.69 & 0.002 \\
\hline Phosphate(mg/dL) & 0.78 & -1.76 to 3.31 & 0.546 \\
\hline LDL-Cho(mg/dL) & 0.39 & -0.032 to 0.11 & 0.283 \\
\hline $\mathrm{Lp}(\mathrm{a})(\mathrm{mg} / \mathrm{dL})$ & 0.09 & -0.007 to 0.18 & 0.04 \\
\hline Diabete Status & -0.48 & -0.002 to 0.07 & 0.86 \\
\hline \multicolumn{4}{|l|}{ Model 2: $\mathrm{R}^{2}=0.46(\mathrm{~N}=131)$} \\
\hline $\operatorname{Age}(y)$ & 0.55 & 0.35 to 0.74 & $<0.001$ \\
\hline Hemodialysis duration(mo) & 0.03 & -0.001 to 0.07 & 0.05 \\
\hline Systolic pressure $(\mathrm{mmHg})$ & 0.44 & 0.27 to 0.60 & $<0.001$ \\
\hline Diastolic pressure $(\mathrm{mmHg})$ & -0.43 & -0.78 to -0.08 & 0.02 \\
\hline Calcium(mg/dL) & 9.11 & 4.06 to 4.16 & $<0.001$ \\
\hline $\mathrm{Lp}(\mathrm{a})(\mathrm{mg} / \mathrm{dL})$ & 0.09 & 0.004 to 0.17 & 0.04 \\
\hline \multicolumn{4}{|l|}{ Model 3: $R^{2}=0.44(N=131)$} \\
\hline Age(y) & 0.53 & 0.34 to 0.72 & $<0.001$ \\
\hline Systolic pressure(mmHg) & 0.43 & 0.26 to 0.60 & $<0.001$ \\
\hline Diastolic pressure $(\mathrm{mm} \mathrm{Hg})$ & -0.46 & -0.81 to -0.11 & 0.01 \\
\hline Calcium(mg/dL) & 10.59 & 5.717 to 15.46 & $<0.001$ \\
\hline $\mathrm{Lp}(\mathrm{a})(\mathrm{mg} / \mathrm{dL})$ & 0.09 & 0.004 to 0.17 & 0.04 \\
\hline
\end{tabular}

Abbreviations: ACAI, aortic calification area index; LDL, low-density lipoprotein.

Table 3. Multivariable Logistic Regression Risk for Aortic Calcification

\begin{tabular}{|c|c|c|c|}
\hline & Odds Ratio & 95\% Confidence interval & $\mathbf{p}$ \\
\hline \multicolumn{4}{|l|}{ Model 1 ( $N=131)$} \\
\hline Age(y) & 1.14 & 1.07 to 1.22 & $<0.001$ \\
\hline Hemodialysis duration(mo) & 1.01 & 1.00 to 1.02 & 0.034 \\
\hline Systolic pressure $(\mathrm{mmHg})$ & 1.08 & 1.03 to 1.14 & 0.002 \\
\hline Diastolic pressure $(\mathrm{mmHg})$ & 0.98 & 0.89 to 1.08 & 0.64 \\
\hline Calcium(mg/dL) & 4.84 & 1.37 to 17.02 & 0.014 \\
\hline Phosphate(mg/dL) & 0.82 & 0.47 to 1.44 & 0.50 \\
\hline LDL-Cho(mg/dL) & 1.01 & 0.99 to 1.02 & 0.54 \\
\hline $\mathrm{Lp}(\mathrm{a})(\mathrm{mg} / \mathrm{dL})$ & 1.02 & 0.99 to 1.04 & 0.15 \\
\hline Diabete Status & 0.78 & 0.23 to 2.68 & 0.69 \\
\hline \multicolumn{4}{|l|}{ Model 2 ( $N=131)$} \\
\hline Age(v) & 1.15 & B1.08 to 1.22 & $<0.001$ \\
\hline Hemodialysis duration(mo) & 1.01 & 1.00 to 1.02 & 0.016 \\
\hline Systolic pressure( $\mathrm{mmHg}$ ) & 1.07 & 1.04 to 1.11 & $<0.001$ \\
\hline Calcium(mg/dL) & 4.78 & 1.39 to 16.41 & 0.013 \\
\hline $\mathrm{Lp}(\mathrm{a})(\mathrm{mg} / \mathrm{dL})$ & 1.02 & 1.00 to 1.04 & 0.14 \\
\hline \multicolumn{4}{|l|}{ Model 3 ( $N=137)$} \\
\hline Age(y) & 1.16 & 1.10 to 1.23 & $<0.001$ \\
\hline Hemodialysis duration(mo) & 1.01 & 1.00 to 1.02 & 0.013 \\
\hline Systolic pressure $(\mathrm{mmHg})$ & 1.07 & 1.04 to 1.11 & $<0.001$ \\
\hline Calcium(mg/dt) & 4.22 & 1.04 to 13.50 & 0.015 \\
\hline
\end{tabular}

Abbreviations: ACAI, aortic calification area index; LDL, low-density lipoprotein.

usually performed annually, which provides an opportunity to assess vascular calcification as well. From the perspective of accuracy, the ACAI has an advantage over the ACI for evaluating abdominal aortic calcification and is also useful for serial observation of changes in calcification.

In the present study, comparison of the risk factors in the $\mathrm{H}$ and L ACAI groups showed significant differences in age, systolic BP, and the serum calcium and Lp(a) levels. Furthermore, multivariate analysis using multivariable linear regression identified age, systolic BP, and the serum calcium and $\mathrm{Lp}$ (a) levels as independent risk factors. On logistic regression analysis, age, duration of dialysis, systolic BP, and serum calcium represented independent risk factors for severe abdominal aortic calcification. Our study results indicate that the serum calcium level is a significant risk factor.

Hyperphosphatemia is a common complication of patients with stage $5 \mathrm{CKD}$. Patients with a serum phosphorus (P) concentration of $>6.5 \mathrm{mg} / \mathrm{dL}(2.1 \mathrm{mmol} / \mathrm{L})$ have an associated relative risk of mortality of 1.27 , and an inability to control serum $\mathrm{P}$ levels leads to serious complications, in- 
cluding renal osteodystrophy and hyperparathyroidism (11).

Control of dietary $\mathrm{P}$ intake and HD are partially effective in reducing the serum $\mathrm{P}$ level, but it is impossible to consistently maintain it within the normal range in HD patients (12). Therefore, most HD patients require a phosphate-binding drug to trap dietary $\mathrm{P}$ and reduce its absorption.

Calcium carbonate $\left(\mathrm{CaCO}_{3}\right)$ is the main phosphate binder used in Japan. It is effective, but a high calcium positive balance with hyperphosphatemia can induce ectopic calcification, especially of blood vessels. The possibility that the oral calcium dose prescribed to patients favors vascular calcification is supported by a recent study by Chertow et al who demonstrated that progression of arterial calcification scores for the aorta and coronary arteries was more rapid in patients receiving calcium-containing phosphate binders than in those taking sevelamer (13). London et al demonstrated that arterial media calcification was closely associated with the duration of HD and calcium-phosphate disorders, including the oral dose of elemental calcium $\left(\mathrm{CaCO}_{3}\right)$ prescribed as a phosphate binder (4). The Kidney Disease Outcome Quality Initiative (K/DOQI) guideline recommends a lower treatment target for serum $\mathrm{Ca}$, as well as for serum $\mathrm{P}$, based on analysis of each patient's outcome (14). Recently, the importance of treating hyperphosphatemia without affecting serum Ca levels has been highlighted, as conventional treatment with $\mathrm{CaCO}_{3}$ results in a high incidence of ectopic calcification. Sevelamer hydrochloride and lanthanum carbonate are non-aluminum- and non-calcium-containing phosphate binders that recently became available in Japan for the management of hyperphosphatemia in patients on HD.

When this study was performed the only phosphate binder was $\mathrm{CaCO}_{3}$, because neither sevelamer nor lanthanum was available in Japan. Furthermore, Cinacalcet has recently become available for the management of secondary hyperparathyroidism, but at the time of this study the only treatment for secondary hyperparathyroidism was vitamin D agents.

Therefore, the patients' calcium levels in this study might be in high balance for maintaining serum $\mathrm{P}$ levels within the recommended range.

\section{Study limitation}

Because this study was a cross-sectional study we can only suggest that ACAI and age, systolic blood pressure and serum calcium level have a significant correlation in HD patients. However, because severe vessel calcification may be associated with mortality or cardiovascular disease in HD patients, we consider that these factors may be the "keys" to preventing the risk for cardiovascular deaths.

We are continuing to investigate the significance of the ACAI and these factors on cardiovascular mortality in HD patients in a cohort study.

More studies regarding optimal BP control, serum cal- cium control and vitamin D control in HD patients are needed.

\section{Conclusion}

Using the ACAI was an accurate and useful method of evaluating abdominal calcification in HD patients. Using this tool, we found that risk factors for abdominal aortic calcification in HD patients include age, systolic BP, and the serum calcium level.

\section{References}

1. Goodman WG, Goldin J, Kuizon BD, et al. Coronary-artery calcification in young adults with end-stage renal disease who are undergoing dialysis. N Engl J Med 18: 1478-1483, 2000.

2. Patient Registration Committee, Japanese Society for Dialysis Therapy. Overview of regular dialysis treatment in Japan. Ther Apher Dial 12: 49-54, 2007.

3. Shigematsu T, Kono T, Satoh K, et al. Phosphate overload accelerates vascular calcium deposition in end-stage renal disease patients. Nephrol Dial Transplant 18 (Suppl 3): iii86-iii89, 2003.

4. London GM, Guerin AP, Marchais SJ, Metivier F, Pannier B, Adda H. Arterial media calcification in end-stage renal disease: impact on all-cause and cardiovascular mortality. Nephrol Dial Transplant 18: 1731-1740, 2003.

5. Shoji T, Emoto M, Shinohara K, et al. Diabetes mellitus, aortic stiffness, and cardiovascular mortality in end-stage renal disease. J Am Soc Nephrol 12: 2117-2124, 2001.

6. Raggi P, Boulay A, Chasan-Taber $S$, et al. Cardiac calcification in adult hemodialysis patients: A link between end-stage renal disease and cardiovascular disease? J Am Coll Cardiol 39: 695-701, 2002.

7. Yokoyama K, Nishioka M, Sakura T, et al. Most patients with coronary artery calcification have no coronary artery stenosis and hyperphosphatemia should be important in reevaluating the $\mathrm{K} /$ DOQI guideline. Ther Apher Dial 10: 101, 2006.

8. Yukawa S, Sonobe M, Tone Y, et al. Prevention of aortic calcification in patients on hemodialysis by long-term administration of vitamin E. J Nutr Sci Vitam 38 (Suppl): S187-S190, 1992.

9. Go AS, Chertow GM, Fan D, McCulloch CE, Hsu CY. Chronic kidney disease and the risks of death, cardiovascular events, and hospitalization. N Engl J Med 351: 1296-1305, 2004.

10. Foley R, Parfrey PS, Sarnak MJ. Clinical epidemiology of cardiovascular disease in chronic renal disease. Am J Kidney Dis 32 (Suppl): S112-S119, 1998.

11. Block GA, Klassen PS, Lazarus JM, Ofsthun N, Lowrie EG, Chertow GM. Mineral metabolism, mortality, and morbidity in maintenance haemodialysis. J Am Soc Nephrol 15: 2208-2218, 2004.

12. Rufino M, de Bonis E, Martin M, et al. Is it possible to control hyperphosphatemia with diet, without inducing protein malnutrition. Nephrol Dial Transplant 13 Suppl 3: 65-67, 1998.

13. Chertow GM, Raggi P, Chasan-Taber S, Bommer J, Holzer H, Burke SK. Determinants of progressive vascular calcification in haemodialysis patients. Nephrol Dial Transplant 19: 1489-1496, 2004.

14. National Kidney Foundation; K/DOQI clinical practice guidelines for bone metabolism and disease in chronic kidney disease. Am J Kidney Dis 42: S1-S201, 2003.

(C) 2010 The Japanese Society of Internal Medicine http://www.naika.or.jp/imindex.html 